Alarcon, Leonardo Gonzalez, Myriam Elizabeth Vaca Rec alde, Mauricio Marcano, and Enrique Marti. "Adaptable Emergency Braking Based on a Fuzzy Controller and a $\mathrm{P}$ redictive Model." 2018 IEEE International Conference on Vehicular Electronics and Safety (ICVES) (Septembe r 2018). doi:10.1109/icves.2018.8519586. 


\section{Adaptable Emergency Braking Based on a Fuzzy Controller and a Predictive Model}

\author{
Leonardo Gonzalez Alarcon \\ Dept. of Cybersecurity and Safety, \\ ICT division \\ Tecnalia $R \& D$ \\ Bilbao, Spain \\ leonardo.gonzalez@tecnalia.com
}

\author{
Myriam Elizabeth Vaca Recalde \\ Dept. of Automotive, \\ Industry and Transportation \\ Tecnalia $R \& D$ \\ Bilbao, Spain \\ myriam.vaca@tecnalia.com
}

\author{
Mauricio Marcano \\ Dept. of Automotive, \\ Industry and Transportation \\ Tecnalia $R \& D$ \\ Bilbao, Spain
}

mauricio.marcano@tecnalia.com

\author{
Enrique Marti \\ Dept. of Automotive, \\ Industry and Transportation \\ Tecnalia $R \& D$ \\ Bilbao, Spain \\ enrique.marti@tecnalia.com
}

\begin{abstract}
This work presents the implementation of an adaptable emergency braking system for low speed collision avoidance, based on a frontal laser scanner for static obstacle detection, using a D-GPS system for positioning. A fuzzy logic decision process performs a criticality assessment that triggers the emergency braking system and modulates its behavior. This criticality is evaluated through the use of a predictive model based on a kinematic estimation, which modulates the decision to brake. Additionally a critical study is conducted in order to provide a benchmark for comparison, and evaluate the limits of the predictive model. The braking decision is based on a parameterizable braking model tuned for the target vehicle, that takes into account factors such as reaction time, distance to obstacles, vehicle velocity and maximum deceleration. Once activated, braking force is adapted to reduce vehicle occupants discomfort while ensuring safety throughout the process. The system was implemented on a real vehicle and proper operation is validated through extensive testing carried out at Tecnalia facilities.
\end{abstract}

Index Terms-ADAS, automated driving, emergency braking, fuzzy logic

\section{INTRODUCTION}

Automated Driving is an extensive and deep research field, it combines mature functionalities that are already implemented in the industry with experimental lines that are still fully open.

Autonomous Emergency Braking (AEB) is an Advanced Driving-Assistance System (ADAS) in a mature technological stage, that has been available in production vehicles for more than a decade. The typical implementation of an AEB detects situations of imminent collision, and actuates over the braking system to stop the car before reaching the obstacle. In those cases where collision is unavoidable, AEB systems mitigate the impact severity because they have proved to be more effective, reducing vehicle speed when compared to unassisted driving. A meta-analysis of the real-world effectiveness of AEB systems [1] shows that this technology led to a $38 \%$ reduction of rear-end crashes.

According to Euro NCAP ${ }^{1}$, the word "Emergency" means that the system will intervene only in a critical situation. The principal motivation for keeping such a low profile is to avoid taking responsibility away from the driver. A hard and sudden

\footnotetext{
${ }^{1}$ Autonomous Emergency Braking -https://www.euroncap.com/en/ vehicle-safety/the-rewards-explained/autonomous-emergency-braking/
}

braking maneuver implies a risk to vehicle occupants but also to any other element in the road. Legal and practical reasons favor systems with slightly reduced effectiveness in normal functioning, but that will never cause an accident because it has misinterpreted a risk situation (false positive).

As vehicles include more sensors and computing power, they also grow in functionalities and intelligence. Multiple independent ADAS eventually need to be coordinated, so that they cooperate rather than interfere, starting a gradual shift from ADAS to Automated Driving systems. In this context, basic ADAS functionalities can evolve from strict use in critical situations to risk mitigation tools that have a higher effectiveness, at the cost of being more intrusive.

A thorough discussion on active safety and collision avoidance methods can be found in [2]. Risk assessment prior to collision has been the object of several studies. As an example, [3] presents a review of the main indicators used in collision avoidance such as time to collision (TTC) and the generalization time-to-X (TTX), along with the most used motion models in automated vehicles. A minimum distance prediction (PMD) to an obstacle is introduced in [4] as an indicator for threat, calculated using dynamic variables from the vehicle. In the work presented in [5] a fuzzy system was implemented to assess risk in order to alert the driver, and for a steering controller to avoid collision. The paper [6] describes a full automatic braking system that uses TTC as a criticality measure. In [7] a brake model is approximated by fitting splines, along with a prediction based on a dynamic model of the system.

In this work, an adaptive AEB system based on a fuzzy controller is presented. The system is based on a tunable predictive model that can be modified to integrate factors such as driver attention (reaction time) or braking efficiency (maximum deceleration, as a combination of road and vehicle factors). The output of this model is fed to a fuzzy controller that adapts braking force dynamically during the AEB maneuver. In scenarios of unavoidable collision and edge cases, this system will behave like a regular AEB system. When risk is high but not critical (there is some operative margin) the system provides a softer output that is equally effective avoiding the crash, improving other factors as the risk of 
indirectly causing an accident or passenger discomfort.

Section II presents the proposal in detail, explaining the predictive brake model and fuzzy controller. The proposed approach has been implemented and tested in a real vehicle, which is presented as the Case Study in section III. Experimental results are shown and discussed in section IV, leading to section $\mathrm{V}$ which contains the conclusions of this work.

\section{PROPOSAL}

In order to implement an adaptive emergency braking system, braking behavior was initially recognized using experimental data from the vehicle. This allows us to model and create a prediction to use in our emergency braking as a safety indicator. A diagram of the proposed designed reasoning is shown in fig. 1.

Two systems were implemented: a critical emergency braking that exerts full braking force is meant to evaluate the validity of our prediction, and an adaptive braking system able to modulate the output signal to the brakes and distributes the force along a longer time. Both systems were designed to work in low speed scenarios on a straight collision path, towards a static obstacle.

\section{A. Brake model}

A first order system is used to represent the deceleration exerted by the brakes (Eq. 1), identifying its parameters in the acceleration data from the vehicle. After reaching maximum deceleration in our model, the acceleration remains constant until the vehicle stops.

$$
G(s)=K \mathrm{e}^{-s t_{d}} \frac{1}{\tau s+1}
$$

The step response in time domain for such a system is presented in Eq. 2. The constant $K$ represents the scaling for the maximum deceleration of the vehicle, while $t_{d}$, is the delay between emergency braking activation and a measurable deceleration from the vehicle. Finally, $\tau$ is the time constant for our system. In order to measure this response, several tests with the real vehicle were performed, and fitted to the eq. 2, which yield a $K \approx 6.1 \mathrm{~m} / \mathrm{s}^{2}, \tau \approx 160 \mathrm{~ms}$ and time delay $t_{d} \approx 250 \mathrm{~ms}$.

$$
f(t)=K\left(1-\mathrm{e}^{-\left(t-t_{d}\right) / \tau}\right)
$$

The starting point of the braking maneuver can be dynamically set to any value of acceleration and speed, in our case, we assumed the latest value provided by a D-GPS receiver with RTK capability as our initial acceleration, speed and position. These initial values allow us to estimate the vehicle's behavior during the $t_{d}$ seconds delay, prior to the activation of the deceleration.

Given the focus in low speed and straight trajectories, the problem can be reduced to a one-dimensional space. Eq. 2 can be rewritten taking into account initial values, yielding eq. 3 where $a_{r e f}$ represents the braking amplitude which can be set from zero to $a_{\max }$. Following integration, initial speed $\left(v_{0}\right)$ and position $\left(x_{0}\right)$ can be also included, assuming a simple constant acceleration model as shown in Eq. 4 and 5. Kinematic models have as advantages its simplicity and low computational costs, and can deliver good results in noncontrol related scenarios, such as trajectory estimation [3].

$$
\begin{aligned}
a(t)= & \left\{t<t_{d}: a_{0}\right. \\
& \left.t \geq t_{d}:\left(a_{r e f}-a_{0}\right)\left(1-\mathrm{e}^{\frac{-\left(t-t_{d}\right)}{\tau}}\right)+a_{0}\right\} \\
v(t)= & \left\{t<t_{d}: a_{0} t+v_{0},\right. \\
& t \geq t_{d}: a_{r e f}\left(t-t_{d}\right)+\tau\left(a_{r e f}-a_{0}\right) \mathrm{e}^{\frac{-\left(t-t_{d}\right)}{\tau}} \\
& \left.+a_{0} t_{d}+v_{0}-\tau\left(a_{r e f}-a_{0}\right)\right\} \\
x(t)= & \left\{t<t_{d}: \frac{a_{0} t^{2}}{2}+v_{0} t+x_{0},\right. \\
& t \geq t_{d}: \frac{a_{r e f}\left(t-t_{d}\right)^{2}}{2}-\tau^{2}\left(a_{r e f}-a_{0}\right) \mathrm{e}^{\frac{-\left(t-t_{d}\right)}{\tau}} \\
& +\left(a_{0} t_{d}+v_{0}-\tau\left(a_{r e f}-a_{0}\right)\right)\left(t-t_{d}\right) \\
& \left.+\frac{a_{0} t_{d}^{2}}{2}+v_{0} t_{d}+x_{0}+\tau^{2}\left(a_{r e f}-a_{0}\right)\right\}
\end{aligned}
$$

\section{B. Critical Emergency Braking}

Once brake behavior is modeled, the outcome of an emergency braking action can be estimated. A critical emergency braking design allows the evaluation of the prediction implemented, functioning as a benchmark for our adaptable system. The result of our prediction, is evaluated by finding the correct stop distance to signal the braking starting point. In the critical emergency braking the system actuates at the limit to avoid a collision, meaning $a_{r e f}=a_{\max }$. In order to find this stop distance, the time to stop can be numerically derived whenever $v(t)=0$. At any moment, this estimation of the stop distance is performed given the state variables in the model.

The model was fitted to underestimate the behavior in braking, this pessimistic approach allows our approximation of the system to remain safely within our critical distance to the obstacle. This safety distance is set to 2 meters from the obstacle as shown in fig. 4 . Whenever the stop distance prediction is lower than this safety mark, critical emergency braking its activated, meaning the brakes are fully applied to their maximum.

\section{Fuzzy Emergency Braking}

The behavior exhibit by an emergency braking system is rather hard, which increases the penalty if the system would ever incurred in a false positive activation. This heavily depends on a very accurate obstacle detection and proper classification, nonetheless, another option is to intervene with a greater time-gap in the braking, even though the obstacle might not be perfectly tracked. This behavior would apply a progressive braking force, given the obstacle remains in a collision path or backtrack and return to its normal speed, given the obstacle detection turns to be false. 


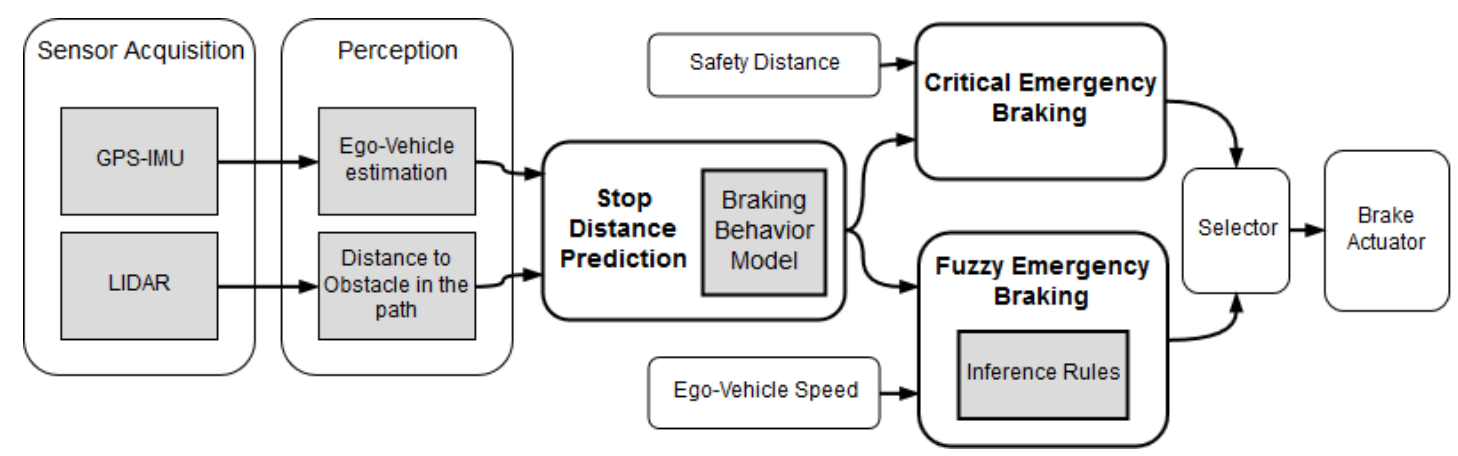

Fig. 1. Emergency Braking logic architecture .

This approach, being more conservative than the previously described critical emergency braking, will performed a gradually increasing brake force, making deceleration smoother. However, this behavior makes it more prone to incur in false positive activation. In order to tuned this trade-off, a fuzzy controller was included, to properly set a series of transitions from soft-braking to hard-braking.

Fuzzy systems have been used in several automotive applications [5], [8] due to its simple tunning and design, based on a set of rules extrapolated from expert driver knowledge. The design of our Fuzzy Emergency brake system makes use of two input variables to modulate the effect on the brake actuator: the stop-distance prediction and the speed of the vehicle, with a single output which is a normalized brake signal, as seen in fig. 2. This signal modulates the amplitude of the step to input the system, which in turn allows us to change the deceleration produced $\left(a_{r e f}\right)$. In the limit, given an obstacle is detected at a critical time, the system will behave at $90 \%$ of the performance in the critical emergency system.

1) Ego-vehicle Speed: The vehicle speed has been taken into account due to its direct relation with crash criticality. In our fuzzy system designed only low speeds (below $40 \mathrm{Km} / \mathrm{h}$ mark) were included. Intuitively, greater speeds means the vehicle has a higher kinetic force to dissipate. Additionally, higher deltas in speed have been related with crash incidents [9]. A higher speed offsets the required braking force for a full stop, increasing the distance needed to brake.

2) Stop Distance: Predicted distance to the obstacle at which the vehicle will stop completely, given the brakes are applied to $100 \%$ immediately. A Stop distance has been used as a criticality indicator in [4]. An accurate estimation of this variable depends upon the obstacle distance perception, and current kinematic variables.

The input function consisted of three classes for low, mid and high distance prediction, where a low distance directly translates into a saturated output. This saturated behavior occurs slightly below the 2 meters range, which guarantees the system will behave similarly to the critical emergency braking design, although with a $90 \%$ performance. This allows the system to be easily tuned for comfort while maintaining withing the safety constraints design already embedded in our critical emergency system.

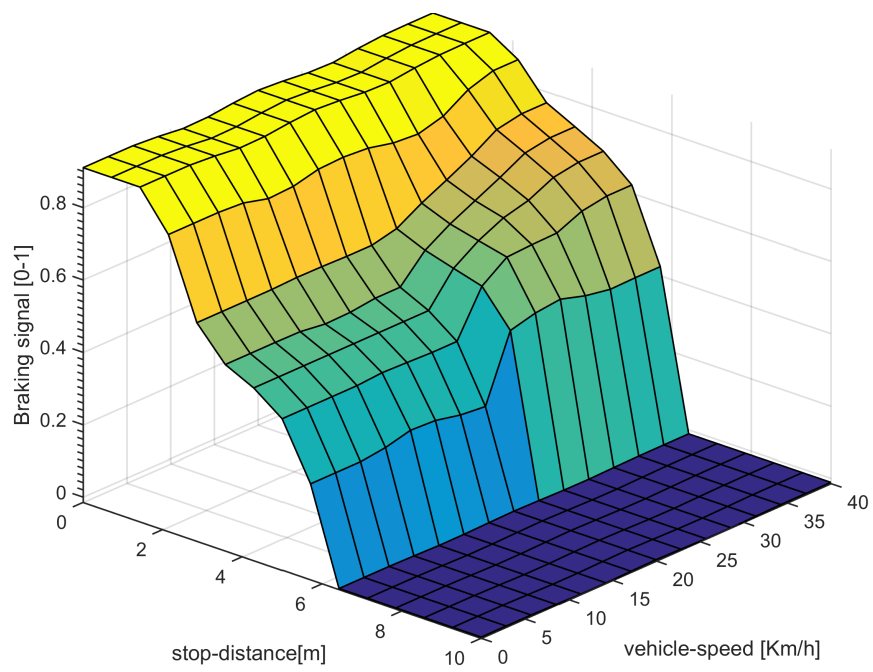

Fig. 2. Output surface for the fuzzy inference system.

\section{CASE STUdY}

This section offers a description of the architecture and experimental platform used in this work. First, a general automated driving framework is presented, detailing the main blocks which compose it. In addition, the experimental platform where braking algorithms were implemented is described.

1) Automated Driving General Framework: Provides an abstraction through the use of a modular architecture which allows the main components in the automated driving task to be distributed in a series of blocks. This framework has been used in the past for several automated driving applications such as: validation of longitudinal controllers [10], comparison of lateral controllers [11], overtaking maneuvers [12] and different speed planner approaches [13]. A detailed block description is presented below.

a) Acquisition: This stage manages the communication with the on-board sensors: D-GPS, Inertial Measurement Unit (IMU), LIDAR, camera, vehicle odometry, actuator encoders and others, which provides raw information of the environment and 
vehicle state. The LIDAR used has a range of 12 meters, additionally the D-GPS used has a precision in the $10 \mathrm{~cm}$ range for position, and $0.1 \mathrm{Km} / \mathrm{h}$ for speed.

b) Perception: This block is in charge of processing all the raw data coming from the acquisition layer and generates useful information for the vehicle road planning: free route recognition, ego-vehicle estimation, obstacle detection and classification. In the work presented, the LIDAR information is processed and obstacles inside a predefined route are extracted.

c) Decision: This module decides the dynamic behavior of the vehicle, consisting of the following three consecutive steps: global, local and behavioral planner. The global planner is responsible to make a first approximation of the road map. Local planner optimizes the created path through different parameters like minimization of curvature, acceleration and jerk. Finally, the behavioral planner adjust the trajectory to overcome dynamic events. An emergency braking represents a contribution to this block, particularly to its behavioral stage.

d) Control: receives the path to be followed by the vehicle from the decision block, and is responsible for tracking the route at a specific speed. It also delivers the control signal to the vehicle actuators. In this work a fuzzy controller is utilized to maintain a reference speed, and additionally in our implementation of emergency braking to modulate the braking behavior.

e) Actuators: the control outputs are transformed to real action signals and move the vehicle actuators through the use of scaling and shifting (throttle, brake, and steering wheel).

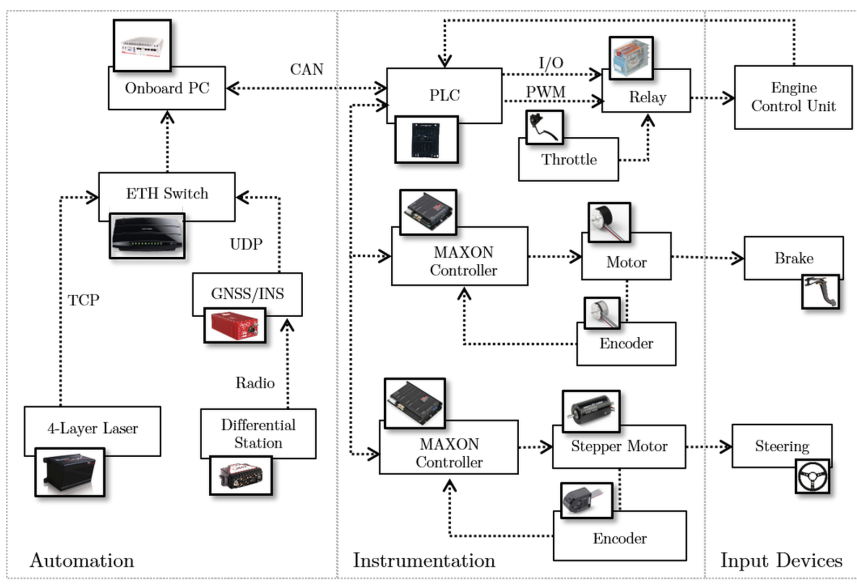

Fig. 3. Instrumentation and automation of a Renault Twizy

2) Real Test Platform: Experimental testing has been carried out on a Renault Twizy 80, a 4-wheel electric vehicle that reaches a maximum speed of $80 \mathrm{Km} / \mathrm{h}$.
As shown in fig. 3, the three main relevant systems are steering, throttle and brake, which are instrumented to allow automated control of the vehicle. These actuators are controlled through a CAN bus network by a Programmable Logic Controller (PLC). The Engine Control Unit (ECU) is operated by the PLC or the throttle pedal selectively. Apart from that, the break pedal is controlled by a rotation motor that pulls or releases the mechanism through a steel wire. Additionally, the steering wheel is controlled by a $250 \mathrm{~W}$ brushless motor attached to an encoder to obtain position feedback. Finally, an onboard computer is in charge of running the algorithms for our automation approach.

\section{EXPERIMENTAL RESULTS}

The fuzzy emergency braking was tested along a critical alternative to control and compare the system with similar commercially available systems, and to evaluate our prediction. The braking performance was evaluated empirically for our real test vehicle, and fitted with the model presented in chapter II, which prove sufficient for a low speed estimation of future vehicle behavior, as can be seen in fig. 5 .

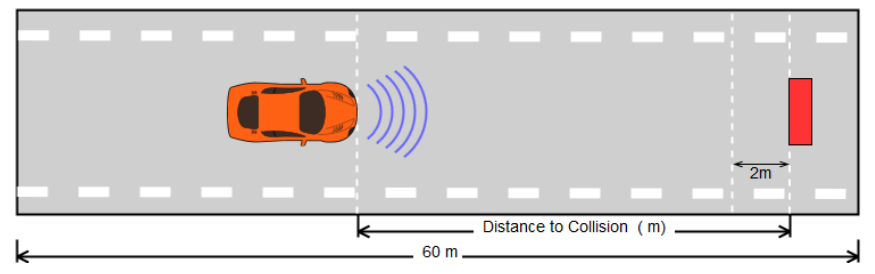

Fig. 4. Diagram of test performed in straight road. The Red-box signals an obstacle, with a 2 meters safety distance.

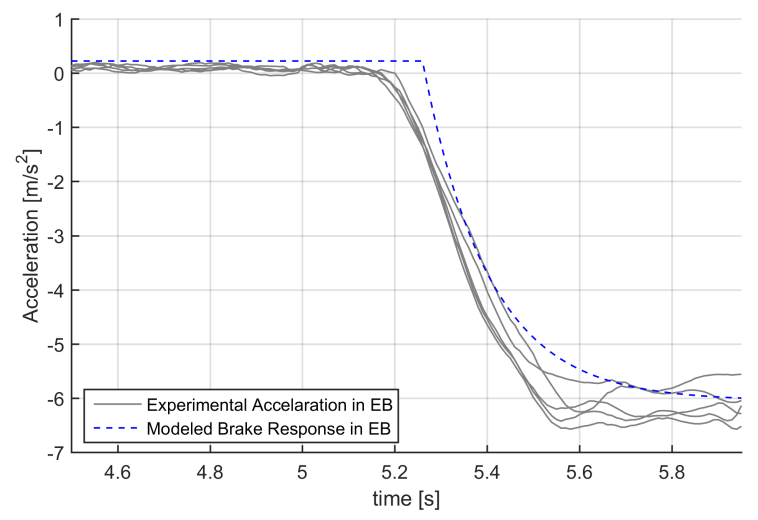

Fig. 5. The modeled response is shown against several empirical tests for Emergency Braking.

The braking controller focuses in low speed performance, testing was conducted at $15 \mathrm{Km} / \mathrm{h}$ and $20 \mathrm{Km} / \mathrm{h}$ in a straight $80 \mathrm{~m}$ test track. A static obstacle was introduced in the path of the vehicle as shown in the fig. 4 and the vehicle remained in automated mode for the entire duration of the tests.

The prediction estimates stop distance as seen in fig. 6, this estimation surpasses the 2 meters limit initially, due to a 
discrepancy on the acceleration model, which is caused by the time delay in the activation and makes the system believe it has missed the mark, even though emergency braking has already started. Nonetheless after deceleration is effectively applied, the prediction becomes optimistic and eventually converges to the distance-to-obstacle when the vehicle is fully stopped.

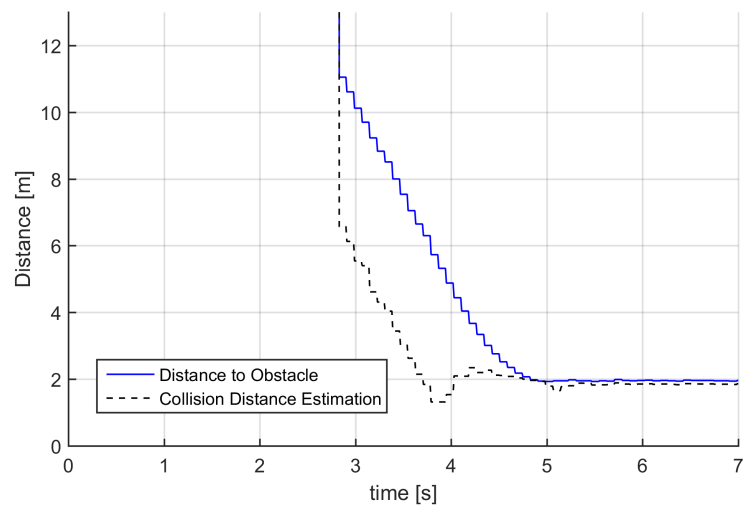

Fig. 6. Stopping distance against direct measurements from LIDAR sensor.

A critical emergency braking was considered whenever the prediction implemented crossed a certain safety value while in the fuzzy approach, the stop-distance value to start braking is close to the 6 meters as seen in fig. 2. It can be seen in fig. 7 , that this difference triggered the braking signal at a more conservative time in the fuzzy approach for both speeds tested.

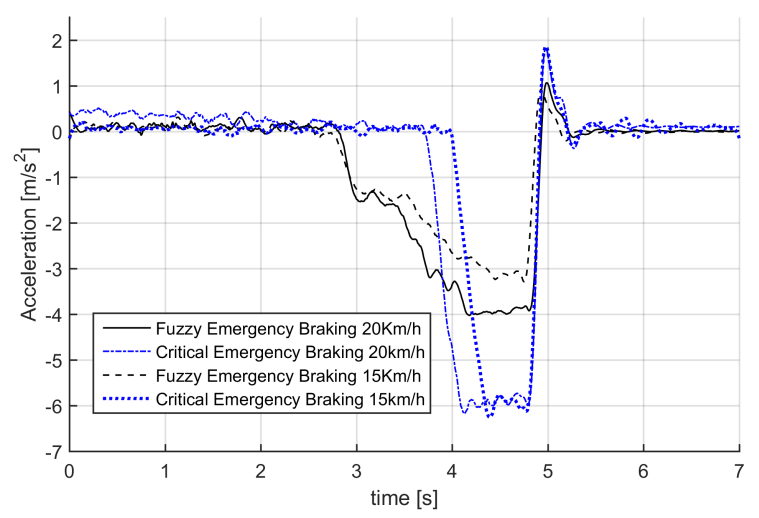

Fig. 7. Deceleration for emergency and fuzzy braking at different speeds.

Additionally a delta between the starting time for different speeds is to be expected, and it can be clearly noted for the emergency braking scenario in fig. 7. However, in the case of fuzzy braking, this difference is almost negligible due to the maximum distance seen by the sensors; at 12 meters of view, the stop distance was below the mark of activation for our fuzzy system.

In fig. 8, the adaptive behavior of the system in relation with the distance can be appreciated. An evolution of the braking signal for the fuzzy braking can be seen along a reduction in speed. As expected, the fuzzy system increased braking force gradually, until the vehicle stopped, based on our stop-distance prediction. In the same figure, it can also be seen how the vehicle to obstacle distance remain in the neighborhood of 2 meters.
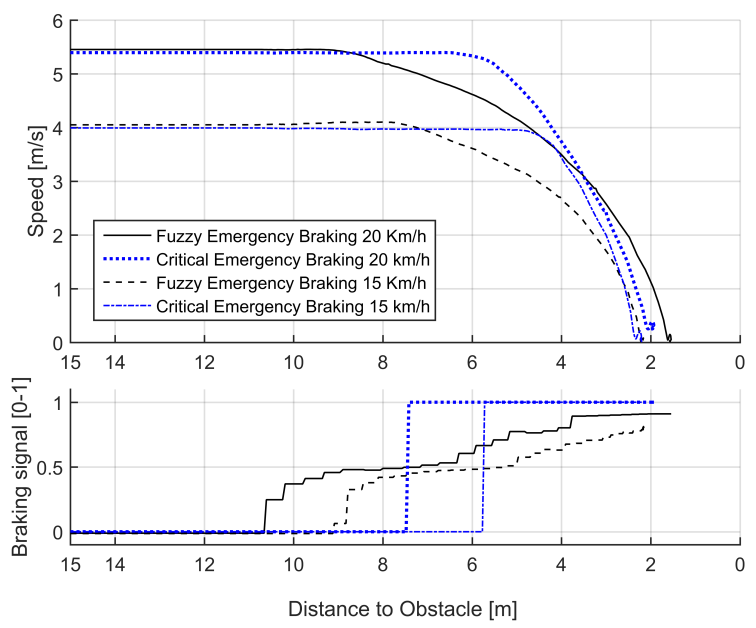

Fig. 8. Vehicle speed is displayed against distance to obstacle, along activation signals.

\section{A. Comparison/Discussion}

In the tests performed, the adaptive system implemented with a fuzzy controller was tested along a critical emergency approach with a fix safety boundary. In both cases, collision was prevented and the vehicle stopped close to the 2 meters mark. In the case of critical system, braking distance to obstacle remained closer to this safety constraint, given the modeled response was a more appropriate approximation of the prediction implemented.

In the case of fuzzy emergency braking, the system engage at $90 \%$ of the braking force at a distance around the 1.6 meters mark. Performance is similar to the emergency braking when evaluating distances to obstacle, the main difference being the deltas in acceleration are greatly reduced, at the expense of an earlier activation.

This early activation supposes a maximum deceleration decrease, along a smaller delta throughout the maneuver. The critical system exerts full deceleration force which for our vehicle is equivalent to $6.1 \mathrm{~m} / \mathrm{s}^{2}$, as soon as the brake actuator engages, while the fuzzy system modulates and distributes this force. In our fuzzy approach the initial deceleration is at the $2 \mathrm{~m} / \mathrm{s}^{2}$ mark, and by the time it reaches maximum, increases to around $4 \mathrm{~m} / \mathrm{s}^{2}$.

Additionally, it should be noted that even though the tests were performed on an automated vehicle, if manual braking were ever to occurred, the stop distance calculation will reestimate and intervention will be delayed, provided the vehicle is not in a collision path anymore.

\section{CONClusion And Future Works}

This work presents and analyzes a fuzzy emergency braking system designed for low speed scenarios. Additionally 
a critical emergency braking was also implemented to test the feasibility of the prediction and a comparison of both systems was conducted. A one dimensional constant acceleration model is used along with a brake model to estimate the deceleration behavior, which approximates the real system response in order to estimate future distance to stop available. Both systems, the fuzzy and critical emergency braking make use of this simplified prediction.

The performance of both critical and fuzzy braking systems in low speed scenarios is similar regarding the distance to obstacle when stopped, while the fuzzy system activation occurs at a much earlier point (around a second before). The maneuver was completed with a much lower maximum deceleration in the case of the fuzzy system, while the critical emergency braking acted in the limit and was faster at stopping the vehicle. Considering the case of a false positive behavior, the deceleration observed in the fuzzy system at an early stage is close to $33 \%$ of the one presented in the critical system, making it easier to return and correct the maneuver. At its maximum, the fuzzy has a peak acceleration of $66 \%$ respect to the one showed by the critical study, making it more comfortable for passengers and less detrimental to the overall control of the vehicle, while maintaining safety.

This implementation provides a first approach towards predictive behavior in obstacle avoidance. In future works, increasing the predictive behavior complexity is a must and could include models adaptable to a variety of scenarios, such as driver behavior considerations, different maneuvers, changes in the environment, and less constrained scenarios with higher speeds in multiple road configurations. The development of this abstraction of layer of prediction allows the incorporation of information from different systems and sensors, with ease into the decision process. The current implementation while using a constraint scenario and a simplified model for prediction, proves satisfactory for an emergency braking at low speed, allowing the simple verification of critical braking to test the limits of the system which allowed the design of a tunable controller to improve over it.

\section{ACKNOWLEDGMENT}

This project has received funding from the Electronic Component Systems for European Leadership Joint Undertaking under grant agreement No 692480. This Joint Undertaking receives support from the European Unions Horizon 2020 research and innovation programme and Germany, Netherlands, Spain, Austria, Belgium, Slovakia.

\section{REFERENCES}

[1] B. Fildes, M. Keall, N. Bos, A. Lie, Y. Page, C. Pastor, L. Pennisi, M. Rizzi, P. Thomas, and C. Tingvall, "Effectiveness of low speed autonomous emergency braking in real-world rear-end crashes," Accident Analysis and Prevention, vol. 81, pp. 24-29, aug 2015.

[2] J. Jansson, Collision Avoidance Theory with Application to Automotive Collision Mitigation. $\mathrm{PhD}$ thesis, Linkping Studies in Science and Technology, 2005.

[3] S. Lefèvre, D. Vasquez, and C. Laugier, "A survey on motion prediction and risk assessment for intelligent vehicles," ROBOMECH Journal, vol. 1, p. 1, jul 2014.
[4] A. Polychronopoulos, M. Tsogas, A. Amditis, U. Scheunert, L. Andreone, and F. Tango, "Dynamic situation and threat assessment for collision warning systems: The euclide approach," in Intelligent Vehicles Symposium, 2004 IEEE, pp. 636-641, IEEE, 2004.

[5] V. Milanés, J. Pérez, J. Godoy, and E. Onieva, "A fuzzy aid rear-end collision warning/avoidance system," Expert Systems with Applications, vol. 39, no. 10, pp. 9097-9107, 2012.

[6] E. Coelingh, A. Eidehall, and M. Bengtsson, "Collision warning with full auto brake and pedestrian detection-a practical example of automatic emergency braking," in Intelligent Transportation Systems (ITSC), 2010 13th International IEEE Conference on, pp. 155-160, IEEE, 2010.

[7] A. L. Rosado, S. Chien, L. Li, Q. Yi, Y. Chen, and R. Sherony, "Certainty and critical speed for decision making in tests of pedestrian automatic emergency braking systems," IEEE Transactions on Intelligent Transportation Systems, vol. 18, no. 6, pp. 1358-1370, 2017.

[8] D. F. Llorca, V. Milanés, I. P. Alonso, M. Gavilán, I. G. Daza, J. Pérez, and M. Á. Sotelo, "Autonomous pedestrian collision avoidance using a fuzzy steering controller," IEEE Transactions on Intelligent Transportation Systems, vol. 12, no. 2, pp. 390-401, 2011.

[9] H. C. Joksch, "Velocity change and fatality risk in a crash-a rule of thumb.," Accident; analysis and prevention, vol. 25, pp. 103-4, feb 1993.

[10] M. Marcano, J. A. Matute, R. Lattarulo, E. Martí, and J. Pérez, "Low speed longitudinal control algorithms for automated vehicles in simulation and real platforms," Complexity, vol. 2018, 2018.

[11] R. Lattarulo, J. Pérez, and M. Dendaluce, "A complete framework for developing and testing automated driving controllers," IFACPapersOnLine, vol. 50, no. 1, pp. 258-263, 2017.

[12] R. Lattarulo, M. Marcano, and J. Pérez, "Overtaking maneuver for automated driving using virtual environments," in International Conference on Computer Aided Systems Theory, pp. 446-453, Springer, 2017.

[13] R. Lattarulo, E. Martí, M. Marcano, J. Matute, and J. Peréz, "A speed planner approach based on bzier curves using vehicle dynamic constrains and passengers comfort," in The International Symposium on Circuits and Systems, IEEE, 2018. 